\title{
Background beliefs in Bayesian inference
}

\author{
JONATHAN ST. B. T. EVANS and SIMON J. HANDLEY \\ University of Plymouth, Plymouth, England \\ DAVID E. OVER \\ Sunderland University, Sunderland, England \\ and \\ NICHOLAS PERHAM \\ Cardiff University, Cardiff, Wales
}

\begin{abstract}
We report five experiments in which the role of background beliefs in social judgments of posterior probability was investigated. From a Bayesian perspective, people should combine prior probabilities (or base rates) and diagnostic evidence with equal weighting, although previous research shows that base rates are often underweighted. These experiments were designed so that either piece of information was supplied either by personal beliefs or by presented statistics, and regression analyses were performed on individual participants to assess the relativeinfluence of information. We found that both prior probabilities and diagnostic information significantly influenced judgments, whether supplied by beliefs or by statistical information, but that belief-based information tended to dominate the judgments made.
\end{abstract}

An important area of human judgment concerns the evaluation and testing of hypotheses. Typically, this occurs under conditions of uncertainty, so that statistical considerations come into play. Although expert evaluation of hypotheses is sometimes assisted by formal statistical procedures, it quite frequently is left to intuition. For example, doctors typically make diagnosticjudgments on the available evidence, without recourse to formal statistics. Similarly, legal decisions, such as those of guilt and innocence by courtroom juries, are unaided by formal statistics, as are many personnel selection decisions and a host of other judgments of real-world importance. It is therefore of great importance for psychologists to understand the nature of such judgments, the biases that may arise, and the conditions under which judgments will be more or less accurate.

In this paper, we concentrate on the area of Bayesian inference. When two alternative hypotheses are under consideration, there are normally two kinds of statistical information that may help to decide between them. First, there is prior probability, or base rate. This refers to the degree of belief that we have about the hypotheses before considering any specific new piece of evidence. However, if such evidence is available, it may provide us with diagnostic information that discriminates between the alternatives.

This research was supported by Grant R000222085from the Economic and Social Research Council of the United Kingdom. The authors thank Ian Dennis for advice on statistical matters. Correspondence concerning this article should be addressed to J. St. B. T. Evans, Centre for Thinking and Language, Department of Psychology, University of Plymouth, Plymouth PL4 8AA, England (e-mail: jevans@ plymouth. ac.uk).

—Accepted by previous editorial team
These two kinds of information are linked by Bayes's theorem, which may be expressed for discrete alternative hypotheses as follows:

$$
\frac{P(\mathrm{H} \mid \mathrm{D})}{P(\overline{\mathrm{H}} \mid \mathrm{D})}=\frac{P(\mathrm{H})}{P(\overline{\mathrm{H}})} \times \frac{P(\mathrm{D} \mid \mathrm{H})}{P(\mathrm{D} \mid \overline{\mathrm{H}})} .
$$

This equation should be read from left to right as posterior odds $=$ prior odds $\times$ likelihood ratio.

The posterior odds are the odds in favor of one hypothesis $(\mathrm{H} 1, \mathrm{H} 2)$ over the other after viewing some specific evidence or datum (D). This is given by the product of the prior odds (ratio of base-rate probabilities) and the likelihood ratio. The likelihood ratio provides the diagnostic evidence. If the datum is more likely to have occurred with $\mathrm{H} 1$ than with $\mathrm{H} 2$, the evidence is diagnostic and favors $\mathrm{H} 1$. Both base rates and likelihood ratios can favor either hypothesis by varying degrees.

It is hard to contest Bayes's theorem as a normative model for hypothesis evaluation, since it is a direct consequence of the axioms of the probability calculus. However, the theorem does lead to some surprising and counterintuitive conclusions. Consider a medical scenario in which people are being screened for a medical condition. Suppose that a test has a hit rate of $100 \%$ but a false positive rate (FPR) of $0.1 \%$, so that $1 / 1,000$ people who do not have the condition produce a positive test result. This sounds impressive, but the test would actually be of limited value in screening for a rare condition. For example, if the incidence in the population were 1 in 10,000, a person testing positive would still have posterior odds of 10 to 1 against having the condition. Of course, if other evidence were available to reduce the prior probability of the condition to, say, 1 in 100 , the test would be more useful, generating 
posterior odds of 10 to 1 in favor of someone testing positive's having the condition.

There have been many claims in the judgment literature that people neglect or underweight base-rate information in Bayesian reasoning, dating from the famous paper of Kahneman and Tversky (1973). One of the original experiments involved a task in which participants were given a brief sketch of an individual, designed to elicit a stereotypical image of an engineer or a lawyer. They found, for example, that a person with a description that is seen as "representative" of an engineer was judged to be more likely to be an engineer, regardless of whether participants were told that he was drawn from a pool of 70 engineers and 30 lawyers or of 30 engineers and 70 lawyers. The base rate stated had a significant but very small influence on judgments and was greatly underweighted relative to the prescriptions of Bayes's theorem. Kahneman and Tversky (1972b) explained the bias as reflecting dependence on the representativeness heuristic, leading to a judgment of probability based on perceived similarity. There have been many subsequent replications and demonstrations of base-rate neglect, using several different tasks.

We can distinguish between a strong base-rate neglect hypothesis in which people ignore base rates and a weak base rate neglect hypothesis in which people underweight base-rate information. In a review of the extensive research literature on the lawyers and engineers problem, Koehler (1996) showed that base rates nearly always influence judgments and argued that the notion of strong base-rate neglect has been oversold, acquiring the status of a myth. However, as a number of commentators on his paper have argued (Edgell, Roe, \& Dodd, 1996; Hamm, 1996; Keren \& Thijs, 1996; Thompsen \& Borgida, 1996), there is good evidence in the literature for the weak hypothesis of baserate neglect. In many situations, people $d o$ underweight base-rate information, including cases of expert decision making where it is vitally important. The important issue, according to these commentators, is to discover the factors that influence the extent to which people give weight to base rates in their judgments. Keren and Thijs (1996) further comment that a good approach

would be to apply a regression analysis in which the logarithmic transformation of the prior odds (i.e., base rates) and likelihood ratios are used as independent variables. The advantage of this approach is that the relative weight of base rates and likelihoodscan be directly estimated .... Such an approach, however, would require the specification of relevant likelihoods (which has not usually been done).

In the experiments to be reported in this paper, we followed precisely the strategy suggested by Keren and Thijs (1996) and will report the results of a series of such regression analyses, carried out on the judgments of individual participants.

In the Bayesian framework, the rational judge is supposed to combine information about prior probabilities with the diagnostic information available from some evidence.
Typical experiments claiming base rate neglect have supplied the prior probabilities in the form of explicit base rate statistics. However, in other literature, such as that on belief bias in deductive reasoning (Evans \& Over, 1996, chap. 5) and the study of stereotypes in the social cognition literature (Funder, 1996), an apparently opposite bias is demonstrated. That is to say, people are accused of bias because they introduce prior belief into cognitive tasks where it is formally irrelevant. A number of authors have envisaged the influence of implicit pragmatic processes that interfere with conscious efforts to reason according to the instructions (Evans \& Over, 1996; see, also, Kahneman \& Frederick, in press; Reber, 1993; Stanovich, 1999). Kahneman and Frederick, for example, argue that the base-rate fallacy will result from a heuristic that substitutes representativeness for probability, unless participants succeed in inhibiting this with conscious reasoning.

We take the view that the powerful influences attributed to representativeness are pragmatic, reflecting the realworld beliefs of the participant, such as stereotypicalbeliefs about the kinds of people that lawyers and engineers are. The pragmatic influence of prior belief seems to dominate the explicit statistical information typically used to communicate prior probabilities via base rates. Would this trend be reversed, however, if (1) the beliefs used to communicate the likelihood ratio were, instead, used to communicate the prior probabilities and (2) diagnostic information were presented statistically?Precisely this comparison is permitted in the experiments to be presented here. We know of one small-scale study (Evans, Brooks, \& Pollard, 1985 ) in which the Bayesian priors were supplied by realworld beliefs and exerted substantial influence on the posterior probability judgments made. There has also been a number of studies reported in the literature (reviewed by Koehler, 1996) in which base rates have been supplied implicitly by experimental training. The general finding is that directly experienced base rates are utilized more than those presented in the standard paradigm, but results can be complex and equivocal (Medin \& Bettger, 1991; Medin \& Edelson, 1988).

The present study extends the investigation of Bayesian reasoning to include the use of prior probabilities supplied by stereotypical belief, rather than experienced base rates as such (see Experiments 4 and 5). It also introduces two methodological innovations: (1) the use of regression analyses on individual participants to measure the relative influence of prior odds and likelihood ratio and (2) the use of precisely the same beliefs to supply likelihood ratios in some experiments and prior odds in others.

\section{EXPERIMENT 1}

Experiment 1 was designed primarily to establish a database of beliefs to support the design of subsequent experiments. The questions concerned membership of student societies in an imaginary university as a function of faculty membership. (In British universities, a faculty is an orga- 
nizational grouping of academic departments representing cognate topics.) This is the kind of topic from which we might expect to elicit stereotypical beliefs about the kinds of people that engineers are, as opposed to social scientists and so on. In addition to providing a database, Experiment 1 also allowed us to look at the relationship between judgments of $P(\mathrm{H} \mid \mathrm{D}), P(\mathrm{D} \mid \mathrm{H})$, and $P(\mathrm{H})$ for a set of hypotheses. If participants base their judgments on intuitions of similarity and representativeness, we might expect to find, as Kahneman and Tversky (1973) did, a high correlation between assessments of $P(\mathrm{H} \mid \mathrm{D})$ (faculty, given society membership) and $\mathrm{P}(\mathrm{D} \mid \mathrm{H})$ (society membership, given faculty). This is because both judgments would derive from the assessment of the degree to which $\mathrm{D}$ is representative of $\mathrm{H}$. However, we also measured estimates of $P(\mathrm{H})$, the relative number of students in each faculty. These base rates were based on personal beliefs rather than presented statistics, so in line with the arguments given above, we would predict an effect of these base rates also on the judgments of posterior probability, $P(\mathrm{H} \mid \mathrm{D})$.

\section{Method}

Participants. Forty undergraduate students of the University of Plymouth participated on a voluntary basis.

Procedure. All the participants were run individually and were told that the experiment involved answering questions regarding the size of memberships of faculties and societies at the University of Utopia. Although fictional, the university was said to be representative of British universities around the country. The four faculties were engineering, social science, arts/humanities, and science. The 20 societies were as follows: classical music, techno music, jazz music, socialist, conservative, liberal democrat, green, parachuting, chess, judo, real ale, cookery, orienteering, drama, dance, choral, table tennis, surfing, U.F.O., and computer.

Materials. Each participant received a booklet that contained three sets of questions. The preliminary instructions indicated that the participants would be asked questions about a fictional university (Utopia) that was representative of universities in Britain. They were told that each student society had 100 members, and on average, an equal number of students from each faculty joined societies. For purposes of the Bayesian parameters, faculties corresponded to hypotheses and society membership to data. One set of questions asked about the probability of belonging to a faculty, given that someone was a member of a particular society. This was the posterior probability judgment, $P(\mathrm{H} \mid \mathrm{D})$. For each of the 20 societies in turn, the participants were asked to indicate the frequency of faculty membership by distributing the 100 society members into the four faculties.

The second set of questions required the participants to decide the probability of being a member of a society, given that a student belonged to a particular faculty $[P(\mathrm{D} \mid \mathrm{H})]$. A different response task was adopted so as not to appear too similar to the measurement of $P(\mathrm{H} \mid \mathrm{D})$ described above. Each of the 20 societies was again con- sidered in turn, but the participants were now asked to judge "how likely it is that students from each of the four faculties would belong to a particular society." For each society, they were asked to make four judgments, one for each faculty, by marking a point on a line labeled $0 \%$ on the left and $100 \%$ on the right.

The order of presentation of the two tasks described was counterbalanced but always followed by a third and final task, which assessed ratings of $P(\mathrm{H})$. The participants were told that the university had 1,000 students in total and were asked to divide these among the four faculties to indicate how many they thought would belong to each.

\section{Results}

The mean estimates for each faculty, averaged across societies, are shown in Table 1, and mean scores, broken down by each faculty and society, are provided in the Appendix. Since average judgments of $P(\mathrm{H} \mid \mathrm{D})$ are taken over all societies, they should normatively correspond to the direct judgments of $P(\mathrm{H})$. That is to say, the total number of students allocated to engineering, say, across all societies should be proportional to the total number of engineers in the university, since students were told that, on average, the same number of students from each faculty joined societies. There is some discrepancy between the two sets of estimates, but both give an approximately equal distribution of students within the four faculties. The column headed $P(\mathrm{D} \mid \mathrm{H})$ can be read as the average number of students from each faculty expected to be in a given society.

Correlations were computed between $P(\mathrm{H} \mid \mathrm{D})$ and $P(\mathrm{D} \mid \mathrm{H})$ across the 20 societies for each of the four faculties, also shown in Table 1 . These correlations were highly significant in all cases. Although the correlations are in line with the predictions of the representativeness hypothesis and similar to some findings reported by Kahneman and Tversky (1973), they are far from conclusive evidence for it. The problem is that the base-rate estimates for the four faculties are roughly equal, so this correlation would also be expected if people were reasoning in a Bayesian manner. In the one faculty that was judged a little larger than others, social science, we see that the correlation was also somewhat lower. In order to investigate whether people were reasoning in a Bayesian manner, we carried out a much more detailed analysis of individuals' responses.

For each individual participant, we have the relevant Bayesian parameters for each of the 20 societies. For example, a participant will have rated both the probability of being an engineer given membership in the chess society, the converse probability, and the overall probability (base rate) of being an engineer in the university. From these we can compute the following components of the Bayesian equation:

Table 1

Mean Estimates (with Standard Deviations) on the Three Tasks in Experiment 1, Expressed as Percentages

\begin{tabular}{|c|c|c|c|c|c|c|c|}
\hline \multirow[b]{2}{*}{ Society } & \multicolumn{2}{|c|}{$P(\mathrm{H} \mid \mathrm{D})$} & \multicolumn{2}{|c|}{$P(\mathrm{D} \mid \mathrm{H})$} & \multicolumn{2}{|c|}{$P(\mathrm{H})$} & \multirow{2}{*}{$\begin{array}{c}\text { Correlation } \\
P(\mathrm{H} \mid \mathrm{D})-P(\mathrm{D} \mid \mathrm{H})\end{array}$} \\
\hline & $M$ & $S D$ & $M$ & $S D$ & $M$ & $S D$ & \\
\hline Engineering & 24 & 8.7 & 47 & 15.1 & 21 & 7.7 & .93 \\
\hline Social science & 25 & 6.2 & 51 & 9.2 & 30 & 9.6 & .66 \\
\hline Arts/humanities & 28 & 9.8 & 55 & 11.6 & 23 & 8.7 & .79 \\
\hline Sciences & 23 & 5.7 & 50 & 12.0 & 26 & 8.5 & .84 \\
\hline
\end{tabular}


posterior odds, $P($ engineer $\mid$ chess $) /(1-P($ engineer $\mid$ chess $)$; prior odds, $P$ (engineer $) /(1-P($ engineer $)$; likelihood ratio, $P$ (chess | engineer) / $P$ (chess $\mid$ nonengineer).

In order to derive the likelihoodratio, we need to estimate $P$ (chess |nonengineer), which can be derived from the estimates that the participant gave for the probability of joining the chess society given membership of the other three faculties.

Having derived the three components for each of the 20 societies, we can perform a multiple linear regression on the ratings of each individual participant, to examine the relative influence of each component. Since Bayes's theorem specifies a multiplicative relationship, we must first compute logarithms of the terms to produce the following additive linear equation: $\log$ (posterior odds $)=\log$ (prior odds) $+\log$ (likelihood ratio). The regression weights computed in this manner tell us whether and to what extent participants make use of each piece of information. ${ }^{1}$ So, for each participant, we compute a multiple linear regression across the 20 societies, regressing log (prior odds) and log (likelihood ratio) onto log (posterior odds). The resulting regression weights indicate the relative influence of the two components on posterior odds. If people are reasoning in a Bayesian manner, the two components should exert an equal weight. We report raw regression weights ( $b$ values), rather than standardized weights (beta values), for two reasons. The $b$ weights are predicted to be equal to one under the normative model, a comparison that is lost by standardizing around observed standard errors. Also, the $b$ weights can be used to make comparisons in the relative use of prior odds (base rates) and likelihood ratios (diagnostic evidence) both within and between experiments in an unbiased manner, as was suggested by Keren and Thijs (1996). Since some of the 40 participants failed to complete every estimate in the booklets, the regression analyses were performed for 32 of them who had complete data sets. The mean regression weights and associated statistics are shown in Table 2.

The regression analyses reveal what the general analyses shown in Table 1 obscure. Posterior probability judgments are strongly predicted by likelihood ratios (diagnostic evidence) and unaffected by prior odds (base rates), with mean regression weights of 0.88 and 0.03 , respectively. Across participants, the $b$ weights for diagnostic evidence are significantly above zero $[t(31)=10.99, p<$
$.001]$ and significantly greater than the weights for base rate $[t(31)=9.26, p<.001]$. Also, $97 \%$ of the participants showed a significant influence of diagnostic evidence in their regression analyses, as compared with only $13 \%$ who were affected by base rate. Hence, the results of Experiment 1 strongly support the representativeness hypothesis and apparently refute our prediction that subjective base rates (based on real prior beliefs) would influence posterior probability judgments. However, we return to the latter issue in Experiments 4 and 5 of the present paper.

\section{EXPERIMENT 2}

In Experiment 2, we asked participants to make posterior probability judgments when base-rate information was presented as explicit statistical information and diagnostic information was wholly implicit, based on prior belief. We used a factorial design in which people were given problems with both high and low explicit base rates and high and low likelihood ratios based on the belief database collected in Experiment 1. On the basis of the work of Kahneman and Tversky (1973) and others, we would expect the implicit diagnosticity of the evidence to have a strong influence on judgments. We would also expect some influence of the presented base rates, in line with the literature. We were particularly interested in the relative weight that the participants would give to base rates and diagnostic information.

Many of the tasks used to demonstrate base-rate neglect do not actually vary the level of base rate presented, although when this has been done, it appears to affect judgments (Heller, Saltzstein, \& Caspe, 1992; Ofir, 1988). For example, Ofir manipulated levels of both base rates and false alarm rates in the famous cabs problem of Kahneman and Tversky (1972a) and found that participants were significantly affected by the base rate presented and the false positive rate, in the normative direction. In Experiment 2 , we used a moderate range of base rate $(10 \%$ vs. $40 \%$, as compared with Ofir's $10 \%$ vs. $90 \%$ ) and combined it with implicit likelihood evidence so that there would be no arithmetic requirement to combine different statistics.

Experiment 2 also included a manipulation to investigate the possible influence of the use of frequency versus probability presentation of key statistics. There are claims

Table 2

Means and Standard Deviations of Regression Weights ( $b$ Values) for Four Experiments, Mean Goodness-of-Fit Estimates $\left(R^{2} \mathbf{a d j}\right)$, and Percentages of Participants Showing a Significant $b$ Weight for each Independent Variable

\begin{tabular}{|c|c|c|c|c|c|c|c|c|}
\hline \multirow[b]{2}{*}{ Experiment } & \multirow[b]{2}{*}{$N$} & \multirow[b]{2}{*}{ Mean $R^{2}$ adj } & \multicolumn{3}{|c|}{$\begin{array}{c}\text { Log Prior Odds } \\
\text { (Base Rates) }\end{array}$} & \multicolumn{3}{|c|}{$\begin{array}{c}\text { Log Likelihood Ratio } \\
\text { (Diagnostic Information) }\end{array}$} \\
\hline & & & $M$ & $S D$ & $\%$ Significant & $M$ & $S D$ & $\%$ Significant \\
\hline 1 & 32 & 0.37 & $0.03 *$ & 0.19 & 13 & $0.88 *$ & 0.45 & 97 \\
\hline 2 & 137 & 0.26 & $0.24 \dagger$ & 0.52 & 27 & $1.97 \ddagger$ & 1.89 & 58 \\
\hline 4 & 35 & 0.50 & $0.21 *$ & 0.42 & 14 & $0.31 \dagger$ & 0.81 & 49 \\
\hline 5 & 47 & 0.32 & $0.43^{*}$ & 0.45 & 38 & $0.19 \dagger$ & 0.77 & 28 \\
\hline
\end{tabular}

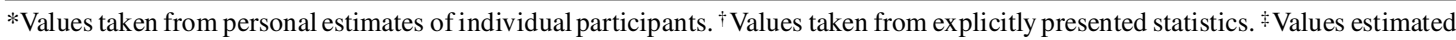
from mean ratings in Experiment 1. 
that the high error rates in Bayesian reasoning often reported in the psychological literature are due to the presentation of such problems in terms of one-case probabilities, rather than as frequencies (Cosmides \& Tooby, 1996; Gigerenzer \& Hoffrage, 1995). Although well established (see Koehler, 1996), the interpretation of such findings is controversial, as is some of the methodology used. Several recent papers have argued that it is not frequency formats per se that facilitate but, rather, the use of problems whose structure cues a mental model facilitating perception of set relationships (Johnson-Laird, Legrenzi, Girotto, Legrenzi, \& Caverni, 1999). When this factor is deconfounded, differences in performance on the two formats disappear (Evans, Handley, Perham, Over, \& Thompson, 2000; Girotto \& Gonzales, 2001; Sloman, 2000). In Experiment 2, the use of frequency or probability formats both in the presentation of base-rate information and in the form of the question asked was varied, without altering any other aspect of the problem information.

In Experiment 2, we predicted an influence of both implicit diagnostic information and explicit base rates. Our regression analyses were employed to discover which information had more influence on the judgments of individual participants. We also predicted, following Evans et al. (2000) and contrary to widespread claims in the literature, that use of frequency or probability presentation formats would not influence judgments in this experiment.

\section{Method}

Participants. One hundred and forty-four students from the University of Plymouth volunteered to take part in the experiment. The participants were run either individually or in small groups. None had taken part in Experiment 1.

Materials. Each participant received a booklet containing an instruction page followed by 32 problems. The preliminary instructions again described the University of Utopia as a typical British university. The participants were told that two of the four faculties each contained $40 \%$ of the students and the other two each had $10 \%$ (the actual faculties named was counterbalanced across participants). The problems presented were very simple and phrased as in the following example:

$40 \%$ of students are in the Engineering faculty.

What is the probability that a member of the Drama society is also in the Engineering faculty, _\%?

The particular societies mentioned were chosen so as to have high or low likelihood- $P(\mathrm{D} \mid \mathrm{H})$ - with regard to the faculty mentioned on the basis of the data collected in Experiment 1. In the example above, membership in the drama society was rated as improbable for engineers, so a judgment of the posterior probability based on representativeness should result in a low estimate.

Design. A $2 \times 2$ factorial design was employed, with presentation format of the base rate and question asked as between-subjects factors, creating four separate groups. In the frequency format condition, the base-rate information was presented as 400 out of 1,000 or 100 out of 1,000 , rather than as $40 \%$ or $10 \%$. This was manipulated independently of question format, which could be in the percentage form shown in the above example or else expressed as _out of _. There were also six subgroups within each of these, which permitted full counterbalancing of the faculties assigned the $40 \%$ or the $10 \%$ base rate.

The within-subjects factors were base rate level $(10 \%, 40 \%)$ and likelihood (high, low). In order to assign high and low likelihoods, we identified the four student societies rated as the most likely and the four rated as the least likely to be joined by students in each of the four faculties in Experiment 1. This provided 16 high- and 16 lowlikelihood problems with differing content. All 32 were used in the problems presented to participants, with half of each type being paired with low and half with high base rates.

\section{Results}

The mean rating in each condition is shown in Table 3. A 2-between- and 2-within-subjects analysis of variance (ANOVA) was performed on the dependentmeasure of posterior probability judgment. The between-subjects factors were base-rate presentation (frequency or percentage) and question type (frequency or percentage), and the withinsubjects factors were likelihood and base-rate level. No significant effects were found of frequency/probability format in either the base rate or the question presented $(F<1$ in both cases). However, the within-subjects manipulations were highly significant in both cases. Judgments were higher for high than for low base rates $[F(1,140)=49.90$, $\left.M S_{\mathrm{e}}=151, p<.001\right]$ and for high than for low likelihoods $\left[F(1,140)=201.60, M S_{\mathrm{e}}=127, p<.001\right]$. There were no significant interactions between the two factors. The additive influence of the two factors can be clearly seen in Figure 1, averaging across the four presentation groups.

The only interaction significant in the ANOVA was that between question format and likelihood $[F(1,140)=6.68$, $\left.M S_{\mathrm{e}}=128, p<.05\right]$. This was a small effect resulting from the fact that, for frequency questions, the effect of high/low likelihood ( $32 \%$ vs. $16 \%$ ) was stronger than that for probability questions ( $31 \%$ vs. $20 \%$ ).

Multiple regression analyses were computed separately for each of 137 individual participants with complete estimates, in a manner similar to that reported for Experiment 1 . The prior odds were taken from the explicit base

Table 3

Mean Posterior Probability Estimates in Each Condition in Experiment 2

\begin{tabular}{|c|c|c|c|c|c|c|c|c|c|}
\hline \multirow[b]{3}{*}{ Condition } & \multicolumn{4}{|c|}{ Frequency Presentation of Base Rate } & \multicolumn{4}{|c|}{ Probability Presentation of Base Rate } & \multirow[b]{3}{*}{ Mean } \\
\hline & \multicolumn{2}{|c|}{ Frequency Question } & \multicolumn{2}{|c|}{$\underline{\text { Probability Question }}$} & \multicolumn{2}{|c|}{ Frequency Question } & \multicolumn{2}{|c|}{ Probability Question } & \\
\hline & Low BR & High BR & Low BR & High BR & Low BR & High BR & Low BR & High BR & \\
\hline Low likelihood & 13.4 & 20.5 & 16.5 & 23.5 & 13.6 & 20.0 & 17.2 & 22.5 & 18.4 \\
\hline High likelihood & 29.8 & 36.7 & 27.9 & 34.2 & 26.2 & 38.1 & 27.2 & 34.3 & 31.8 \\
\hline Mean & 21.6 & 28.6 & 22.2 & 28.9 & 19.9 & 29.1 & 22.2 & 28.4 & \\
\hline
\end{tabular}

Note-BR, base rate. 

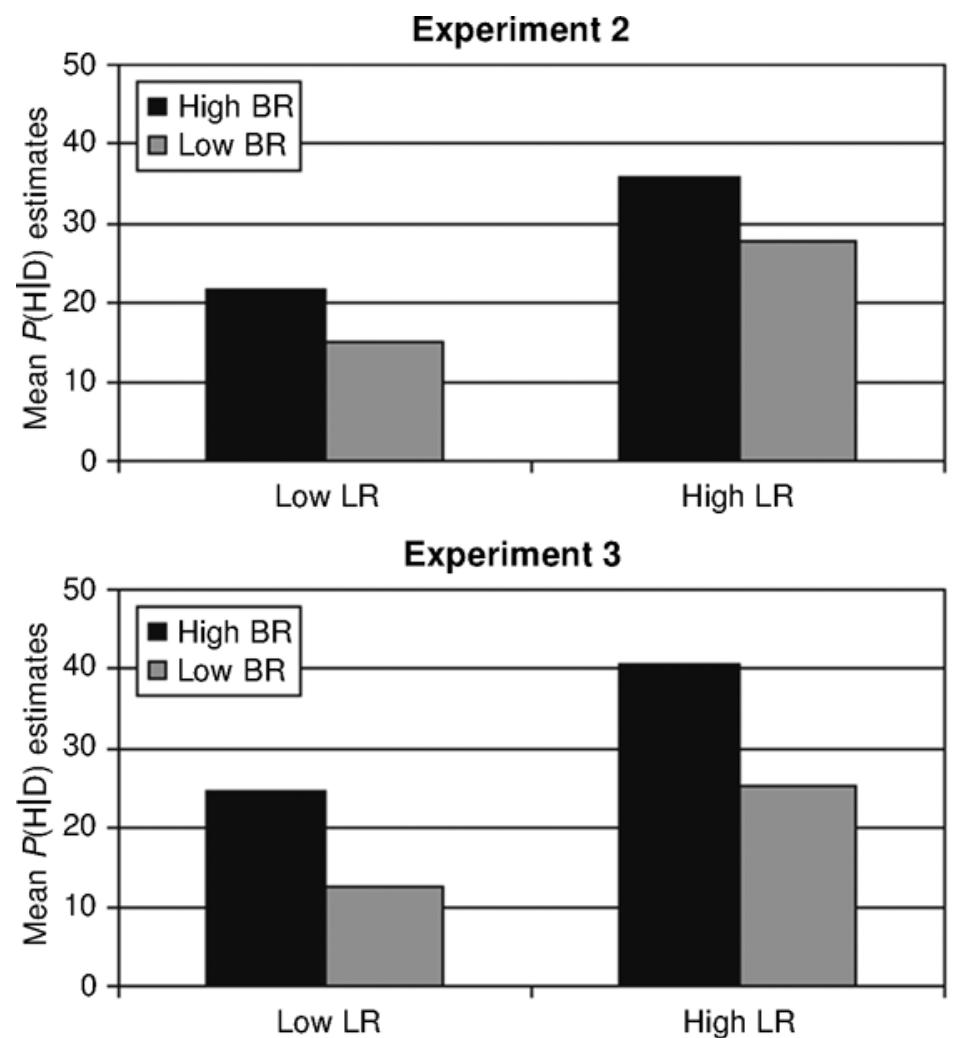

Figure 1. Mean posterior probability estimates in Experiments 2 and 3 broken down by (implicit) likelihood ratio (LR) and base rate (BR).

rates presented. Since the likelihood data were implicit in this experiment, the likelihood ratio for each particular judgment was based on the mean ratings of likelihoods in Experiment 1 for the particular society/faculty combination on each problem. The log values were regressed onto the $\log$ posterior odds computed from the posterior probability ratings given by the participants. The results (see Table 2) provided confirmation of the trends in the ANOVA. Although the participants were influenced by both factors, they were more influenced by implicit likelihoods (mean weighting, 1.97) than by explicit base rates $(0.24)$. Onesample $t$ tests across participants showed that regression weights were significantly above zero for both base rate $[t(136)=5.27, p<.001]$ and likelihood $[t(136)=12.16$, $p<.001]$. Since the normative model requires $b$ weights of one, however, it is clear that many participants are overweighting diagnostic information, as well as underweighting base rates. A related-groups $t$ test confirmed that the regression weights were significantly higher for likelihood than for base rates $[t(136)=10.43, p<.001]$. We should, however, note the high standard deviation for diagnostic $b$ weights in Table 2 and the fact that this weight was significant for only $58 \%$ of the participants. Hence, there was also a good deal of variability between participants in their patterns of judgment.

\section{Discussion}

The failure in Experiment 2 to find any effect of frequency/probability formats in either the presentation of the base rate or the framing of the question provides further support for the claim of Evans et al. (2000) and Girotto and Gonzales (2001) that frequency formats do not in themselves facilitate solution of statistical word problems, contrary to widely cited views to the contrary. The frequency versus probability manipulation is not explored further in this paper, and we will concentrate instead on the other issues raised in our introduction.

We have demonstrated in Experiment 2 that background beliefs exert a strong influence on these probability judgments, since the diagnostic information was entirely implicit. Clearly, our materials can produce the same kinds of effects attributed to representativeness by Kahneman and Tversky (1973). However, we also found a significant effect of explicit base-rate levels on the same judgments. Both the ANOVA and the individual regression analyses performed here showed that explicit base-rate information had a significant influence on judgments. The introduction of regression analyses on individual participants, however, allowed us also to show that the base rate was given significantly less weight in the judgment than was implicit likelihood information, and not equal weight, 
as required by Bayes's theorem. Hence, these findings are consistent with the weak base-rate neglect hypothesis.

Before moving on to the investigation of base rates derived from personal beliefs (Experiments 4 and 5), we first conducted a check on the hypothesis that our participants' use of base-rate information might have been due to the use of a within-subjects design in Experiment 2. Kahneman and Frederick (in press) have argued that within-subjects designs may facilitate the use of explicit reasoning to moderate the influence of unconscious heuristics. This is because the participant is cued that the factor is being varied and is more likely to give it explicit attention. The external validity of between-subjects designs is arguably stronger, since people in the real world will typically lack such cuing of the relevant aspects of their information.

In Experiment 3, we test the hypothesis that attention to base rates will be maintained despite a between-subjects manipulation. We also expect to replicate the effects of implicit diagnosticity through background beliefs.

\section{EXPERIMENT 3}

\section{Method}

Design. The experiment repeated Experiment 2, with two differences. First, the participants were given only the probability (percentage) format in both the base rate and the question asked, and second, the base-rate level was manipulated as a between- rather than a within-subjects factor.

Participants. Sixty undergraduate students of the University of Plymouth participated as volunteers. None had taken part in the previous experiments.

Materials. Each booklet consisted of an instruction page followed by 16 problems. These were the same as those used in Experiment 2 (percentage formats only) and were either high or low base rate throughout, depending on the experimental group.

\section{Results and Discussion}

An ANOVA was performed on the probability judgments, with base rate as a between-groups and likelihood ratio as a within-group factor. As in Experiment 2, the participants gave significantly higher estimates when the base rate was high (mean rating, 33.0) rather than low [18.6; $\left.F(1,58)=17.58, M S_{\mathrm{e}}=321, p<.001\right]$, despite the fact that this was now a between-groups factor and that baserate level was constant throughout any participant's set of problems. The participants again gave higher ratings for the high-likelihoodproblems (32.7) than for the low-likelihood problems [18.9; $\left.F(1,58)=39.21, M S_{\mathrm{e}}=158, p<.001\right]$. The same additive pattern as that for Experiment 2 is apparent in Figure 1.

Since there was no base-rate variation within the set of problems given to each participant, it was not possible to perform the individual regression analyses reported for previous experiments. However, the mean scores suggest that the size of the effect was very similar for both factors and give no clear indication that base rate was underweighted relative to likelihood, as in Experiment 2. This experiment clearly eliminates cuing of base-rate relevance in a within- group design as an explanation of the base rate utilization found in Experiment 2. It fails, however, to add support to the weak base-rate neglect hypothesis suggested by the results of the previous experiment.

\section{EXPERIMENT 4}

In Experiments 2 and 3, the relative influence of implicit diagnostic information was compared with respect to explicit base-rate information, showing substantial influences of both factors. In Experiment 4, we designed a task in which the sources of information were reversed, so that base rates (or prior probabilities) were supplied from background belief and diagnostic likelihoods from presented statistics. We decided to devise a new problem in which precisely the same beliefs would supply the relevant prior probabilities required for the Bayesian inference as were used to supply the diagnostic information in the previous experiments. To show how we did this, we reproduce in full an example of the problems given to the participants in Experiment 4.

The following problems refer to the relationship between faculties and societies within a university. You will be asked to give your best estimate for each problem. Please remember when reading the problems that students can belong to more than one society

A survey was conducted to discover the recreational activities of Engineering students. It was discovered that those Engineering students who belonged to the computer society owned a PC. However, it was also the case that there were Engineering students who did not belong to the computer society but who also owned a PC. Specifically, $5 \%$ of Engineering students who did not belong to the computer society owned PCs. Suppose that a group of Engineering students were randomly selected and asked (a) if they owned a PC and (b) what societies they belonged to. Giving your best estimate, what percentage of those Engineering students who own PCs would you expect to belong to the computer society?__\%

In this problem, the domain was localized to a single faculty, the hypothesis $(\mathrm{H})$ was now the society membership within that faculty, and the datum (D) was ownership of a PC. The posterior probability judgment required, $P(\mathrm{H} \mid \mathrm{D})$, was the probability of (an engineer) belonging to the computer society, given that he/she owns a PC. The likelihood ratio was the hit rate (always fixed at 100\%) divided by the FPR (in this case, $5 \%$ ), which were explicitly stated in the problem. The relevant prior probability (base rate) was therefore the probability of belonging to the computer society, given that the student was an engineer. Thus, the belief that supplied the likelihoodinformation in the previous experiments implicitly provided the base rate in this one.

In Experiment 4, we tested the hypothesis that posterior probability judgments would be significantly and appropriately influenced by (1) base-rate information supplied implicitly by prior belief and (2) explicit presentation of 
FPRs, from which likelihood ratios could be inferred. We used individual regression analyses to estimate and compare the relative influence of each factor.

\section{Method}

Participants. Forty undergraduate students from the University of Plymouth participated on a voluntary basis.

Design and Materials. Each participant received a booklet consisting of an instruction page followed by 16 problem sheets in an individually randomized order. The form of the problems was as shown in the example above, with variations in the faculty, society, and diagnostic information. Faculty/society relationships were chosen so as to produce low implicit base rates for half of the problems and high implicit base rates for the other half. Hit rates were set at $100 \%$, but FPRs varied across problems between the values of 5\%, $15 \%, 30 \%$, and $50 \%$. The higher the FPR, the lower the likelihood ratio favoring the hypothesis stated. Thus, the design comprised $2 \times 4$ within-group factors. There were two versions of each problem type, using differing content. The participants were run in a single large group session.

Finally, the participants were asked to rate all the relevant prior probabilities for the problems that had been presented in the preceding pages. For example, they were asked

What percentage of Engineering students do you think belonged to the computer society _\%

and so on for each of the 16 faculty/society combinations used in the problems. These were presented on a single page in a fixed order.

\section{Results}

The posterior probability judgments were subjected to a $2 \times 4$ within-subjects ANOVA. The mean estimates are shown in Figure 2. In this analysis, the base-rate level was defined a priori (on the basis of Experiment 1 mean ratings) in the way we constructed the materials and without regard for the personal ratings of prior probability taken at the end of the experiment. The base-rate factor had no significant effect $(F<1)$, although there was a significant influence of $\operatorname{FPR}\left[F(3,117)=5.42, M S_{\mathrm{e}}=474, p<.05\right]$. Judgments were influenced in the appropriate direction, with decreasing values being assigned as the FPR increased (see Table 4).

In order to check for an effect of the personal base rates measured at the end of the experiment, a second ANOVA was run reclassifying the 16 problems into the eight highest rated and the eight lowest rated prior probabilities individually for each participant. Since this was no longer crossed with the FPR factor, the analysis was run on the base-rate factor only. Measured in this way, the effect of implicit base rate was statistically significant $[F(1,39)=7.56$, $\left.M S_{\mathrm{e}}=113, p<.01\right]$, with mean ratings of 55.7 for highbase-rate problems and 49.1 for low-base-rate problems.

Multiple regression analyses were conducted on (35) individual participants of $\log$ prior odds and $\log$ likelihood

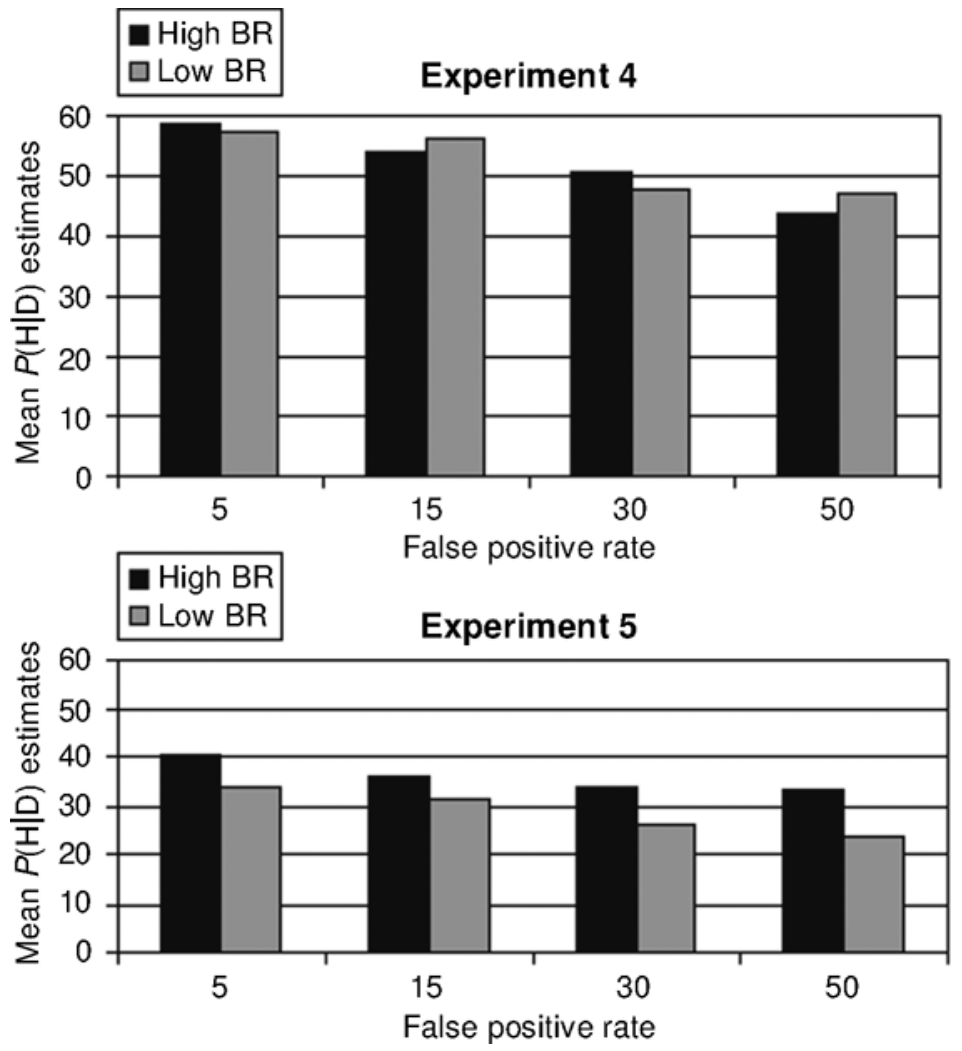

Figure 2. Mean posterior probability estimates in Experiments 4 and 5 broken down by false positive rate and (implicit) base rate (BR). 
ratio onto log posterior odds judgments, as in Experiments 1 and 2. For purposes of this analysis, the prior odds were computed from the individual ratings that each participant gave at the end of the booklet, and likelihood ratios for each problem were derived from the explicit FPR given, being equal to the hit rate (100\%) divided by the FPR. The mean regression weights are shown in Table 2 and were somewhat higher for $\log$ likelihood ratios than for $\log$ prior odds, with $49 \%$ and $14 \%$, respectively, of the participants showing individually significant influences of each factor. Although each measure was significantly above zero (one-sample $t$ values of 2.97 and 2.25 on $34 d f$, significant at $p<.01$ and $p<.05$ for prior odds and likelihood ratios, respectively), a related-groups $t$ test failed to find any significant difference between the mean values of the two indices $[t(34)=0.55]$. Thus, in contrast with Experiment 2 , there is no evidence that base rates (taken from personal ratings) were neglected relative to diagnostic information. Both, however, were underweighted relative to the normatively expected $b$ weights of one.

\section{Discussion}

A clear finding of Experiment 4 is that the participants lowered their posterior probability judgments as the explicitly stated size of the FPR increased. A similar main effect of explicitFPR was reported in experiments on the cabs problem by Ofir (1988). However, Ofir found that that FPR information was utilized only when participants were doubtful about other evidence and was ignored when both stated base rate and hit rate were high. In Experiment 4, hit rate was always $100 \%$, and yet FPR information was still used. Also, there was no interaction between base-rate level and FPR, although it was possible to test for this only with the ineffective a priori base-rate factor. It should also be noted that our experiment differed from Ofir's in that there was no explicit presentation of base rates. It is possible that people find it hard to combine two numerical sources of information and tend to focus on one or the other.

It will be recalled that in Experiment 1, personal base rates measured after other estimates were collected failed to predict posterior probability judgments, even using the sensitive individual regression analyses. The results of Experiment 4 are more encouraging, even though base rates defined a priori, from the mean ratings of Experiment 1 , failed to have a significant effect in the initial ANOVA. Both the further ANOVA based on individual estimates and the regression analyses based on the personal estimates of prior probability showed personal base rates to have a significant influence on judgments in the appropriate direction (higher posterior probability judgments with higher prior probabilities). However, the effect was relatively small, with a mean $b$ weight of 0.21 , as compared with the massive 1.97 found for the effects of implicit belief on diagnostic evidence in Experiment 2.

\section{EXPERIMENT 5}

In our final experiment, we introduced a procedural variation designed to heighten the salience of the personal base rates. We repeated Experiment 4 with one procedural change. The relevant prior probability was rated immediately prior to the problem to which it applied. Arguably, this procedure would force explicit consideration of the base rate. However, the prior probabilities concerned were still personal, based on actual beliefs, rather than on presented statistics.

The hypotheses tested were essentially the same as those specified for Experiment 4. However, we expected our procedural modification to enhance the influence of personal base rates in this experiment.

\section{Method}

Participants. Forty-seven undergraduate students from the University of Plymouth participated on a voluntary basis.

Materials and Procedure. The problems were presented in a booklet with an instruction page followed by 16 problem pages, constructed in the same way as that in Experiment 4, except for one procedural change. Instead of asking the participants to rate prior probabilities for each problem at the end, the relevant judgment was elicited immediately before each problem. For example, the problem might start as follows:

What percentage of Engineering students do you think belong to the computer society? _\%

A survey was conducted to discover the recreational activities of Engineering students ...

The participants were tested in a single large group.

\section{Results and Discussion}

The mean posterior probability estimates for Experiment 5 are shown in Figure 2. It will be noted that the general level of estimates is considerably lower than that in Experiment 4, despite the use of identical problems. This must be due in some way to the instruction to estimate prior probabilities before completing each problem. A $2 \times$ 4 within-group ANOVA was run on the posterior probability judgments, with the factors of base rate (high, low) and FPR on four levels, as in Experiment 4. There were significant main effects of base rate $\left[F(1,46)=19.77, M S_{\mathrm{e}}=\right.$ $284, p<.001]$ and of FPR $\left[F(3,138)=3.09, M S_{\mathrm{e}}=395\right.$, $p<.05$ ], but no interaction between the two $(F<1)$. Note that the base-rate factor in this analysis was taken a priori from the mean ratings in Experiment 1 and was not significant at all in the equivalent analysis reported for Experiment 4 . In the present analysis, there were substantially higher judgments for high base rates (mean, 35.9) than for low base rates (28.7). Once again, the significant effect of FPR indicated that people lowered their probability judgments as the FPR increased.

Inspection of Figure 2 suggests that although both factors influenced the participants' judgments in the correct direction, these influences were rather conservative. That is, we might have expected more pronounced shifts in the means. Individual regression analyses performed for all 47 participants in this experiment confirmed this impression. As the data in Table 2 show, mean $b$ weights for both factors were well below the normatively expected value of one. However, for the first time in this sequence of experiments, we found that the influence of base rate (prior probability) appeared to be stronger than the influence of 
diagnostic evidence (likelihood ratio). Weights on the former factor reached significance for $38 \%$ of the individual participants, as compared with $28 \%$ on the latter. The mean $b$ weight on each was significantly above zero, as assessed by one-sample $t$ tests $[t(46)=6.70, p<.001$, and $t(34)=1.71, p<.05$, one-tailed, respectively]. The difference between the two, however, fell short of significance on a two-tailed test $[t(46)=1.73, p=.09]$.

In view of the similarity of the design of Experiments 4 and 5, we also compared $b$ weights for the two factors between the two experiments. The increased weighting given to base rates in Experiment 5, as compared with Experiment 4 , was significant $[t(80)=2.29, p<.05]$, suggesting that the additional requirement to estimate the relevant base rate at the start of each problem had a clear influence on judgments. This could be seen as an availability effect (Tversky \& Kahneman, 1973), since attention to this information was prompted by the procedure. The reduced $b$ weights for diagnostic information in Experiment 5, however, was not significantly different from those in Experiment $4[t(80)=0.67]$. If weak base-rate neglect is considered to be a neglect of base-rate evidence relative to diagnostic evidence, this clearly is not supported in Experiment 5, where there was a marginally significant trend in the opposite direction.

\section{GENERAL DISCUSSION}

Following the review of Koehler (1996), it is clear that the strong base-rate neglect hypothesis-namely, that people ignore base rates when making posterior probability estimates-is mythical and unsustainable in light of the experimental literature. However, it is also clear from many of the commentaries on Koehler's paper (cited earlier) that the weak base-rate neglect hypothesis is of considerable interest and importance. Although we did not study expert groups in these experiments, the reliable presence of a weak base-rate effect could cause serious biases if present in experts, as several of the commentators pointed out. The relevant prior probabilities for an expert might be derived from statistical information or else from direct realworld experience. For example, a physician might typically draw upon personal experience in assessing the likelihood of common diseases when making diagnostic judgments. It is therefore important to understand the influence of both statistical and belief-based prior probabilities.

The research reported in this paper sheds light on all of these issues. First, we examine the evidence for base-rate neglect. Considering first the conventional ANOVAs on experimentally manipulated factors, we showed in Experiment 2 that posterior probability estimates were significantly higher when the presented base rate was higher and replicated this in Experiment 3 with the base-rate factor manipulated between subjects. The same experiments showed a substantial influence of implicit diagnosticity based on background beliefs, with materials constructed from the mean estimates of Experiment 1. In Experiments
4 and 5 , the same background beliefs provided the baserate information implicitly, whereas diagnostic information was presented by explicit statistics. Again, ANOVAs showed significant influences of both factors, although this was only observed for base rates in Experiment 4 when the data were split by the post hoc individual estimates of each participant, rather than the a priori values derived from Experiment 1.

On the basis of these analyses alone, we could reject (if we had needed to) the strong base-rate neglect hypothesis but would find it difficult to assess evidence for weak base-rate neglect. This is where our individual regression analyses come into play. A word of caution should be expressed about these, however. As is shown in Table 2, we were obliged to use three different sources to estimate the values of the independent variables in these experiments. Where explicit statistical information was presented, these values were used. Where personal estimates were available for each participant for implicit values, these were used. In one case (Experiment 2, likelihood ratios), we estimated the implicit value from the mean ratings of Experiment 1 . This might be expected to underestimate the influence of this factor for individual participants. Despite this, implicit diagnosticity had substantially more influence than did explicit base rates in this experiment, in line with the weak base-rate neglect hypothesis. Background beliefs affecting diagnosticity were also given excessive weight, relative to the normative model, in line with the representativeness hypothesis. Unfortunately, individual regressions were not possible for Experiment 3, where the ANOVA suggested a substantially stronger influence of base rates, despite the between-subjects design.

Weak base-rate neglect is not supported in the findings of Experiments 4 and 5, where base rates were personal and diagnostic information was statistical. In fact, we have marginal evidence in Experiment 5 that prior probabilities exert more weight than does statistical evidence in the judgments of individual participants, when the procedure prompted availability of this information. This base-rate overuse is consistent with the observation in other literature that participants tend be to be biased in the direction of being influenced too much by prior belief (Evans \& Over, 1996; Funder, 1996; Nelson, Biernat, \& Manis, 1990). It could also be interpreted as a vividness effect (Nisbett \& Ross, 1980; Taylor \& Thompson, 1982), in which personal beliefs carry more impact than does pallid statistical information.

In addition to these main findings, our research has also shed some further light on the debate about the use of frequency and probability formats in the presentation of quantitative word problems. Our results do not support the claim that base-rate neglect simply disappears when problems are expressed in terms of frequencies and that this bias is then neatly replaced by adaptive judgments (Cosmides \& Tooby, 1996; Gigerenzer \& Hoffrage, 1995). Experiment 2 supplies direct evidence (in addition to that of Evans et al., 2000, and Girotto \& Gonzales, 2001) that fre- 
quency formats as such have no effect, and our overall results imply that weak base-rate neglect is a real and subtle bias that requires further serious study.

In summary, our research supports the conclusion that utilization of base rates is a complex matter, dependent on both the nature of the information with which participants reason and the method by which it is presented to them. Our five experiments include one (Experiment 2) in which there is clear evidence of weak base-rate neglect and one (Experiment 5) in which there is some evidence of overuse of base rates relative to diagnostic evidence. These findings go some way to resolving the apparently paradoxical conclusions of the literature on base rates and on stereotypes (Funder, 1996). Although intuitions based on personal belief and prior knowledge may be moderated by conscious reasoning about explicit statistics, the evidence of our experiments suggests that intuition will still tend to dominate overall.

\section{REFERENCES}

Cosmides, L., \& Tooby, J. (1996). Are humans good intuitive statisticians after all? Rethinking some conclusions from the literature on judgment under uncertainty. Cognition, 58, 1-73.

Edgell, S. E., Roe, R. M., \& Dodd, C. H. (1996). Base rates, experience, and the big picture. Behavioral \& Brain Sciences, 19, 21.

Evans, J. St. B. T., Brooks, P. G., \& Pollard, P. (1985). Prior beliefs in statistical inference. British Journal of Psychology, 76, 469-477.

Evans, J. St. B. T., Handley, S. H., Perham, N., Over, D. E., \& Thompson, V. A. (2000). Frequency versus probability formats in statistical word problems. Cognition, 77, 197-213.

Evans, J. St. B. T., \& Over, D. E. (1996). Rationality and reasoning. Hove, U.K.: Psychology Press.

Funder, D. C. (1996). Base rates, stereotypes, and judgmental accuracy. Behavioral \& Brain Sciences, 19, 22-23.

Gigerenzer, G., \& Hoffrage, U. (1995). How to improve Bayesian reasoning without instruction: Frequency formats. Psychological Review, 102, 684-704.

Girotto, V., \& GonZales, M. (2001). Solving probabilistic and statistical problems: A matter of information structure and question form. Cognition, 78, 247-276.

Hамм, R. (1996). Physicians ignore base rates, and it matters. Behavioral \& Brain Sciences, 19, 25-26.

Heller, F. H., Saltzstein, H. D., \& Caspe, W. B. (1992). Heuristics in medical and non-medical decision making. Quarterly Journal of Experimental Psychology, 44A, 211-235.

Johnson-Laird, P. N., Legrenzi, P., Girotto, V., Legrenzi, M. S., \& CAVERnI, J. P. (1999). Naive probability: A mental model theory of extensional reasoning. Psychological Review, 106, 62-88.

Kahneman, D., \& Frederick, S. (in press). Representativeness revisited: Attribute substitution in intuitive judgment. In T. Gilovich, D. W. Griffin, \& D. Kahneman (Eds.), Heuristics and biases: The psychology of intuitive judgment. New York: Cambridge University Press.

Kahneman, D., \& Tversky, A. (1972a). On prediction and judgment. ORI Research Monograph, 12.

Kahneman, D., \& Tversky, A. (1972b). Subjective probability: A judgment of representativeness. Cognitive Psychology, 3, 430-454.
Kahneman, D., \& Tversky, A. (1973). On the psychology of prediction. Psychological Review, 80, 237-251.

Keren, G., \& Thiss, L. J. (1996). The base rate controversy: Is the glass half-full or half-empty? Behavioral \& Brain Sciences, 19, 26.

KoeHLer, J. J. (1996). The base rate fallacy reconsidered: Descriptive, normative and methodological challenges. Behavioral \& Brain Sciences, 19, 1-53.

Medin, D. L., \& Bettger, J. (1991). Sensitivity to changes in base-rate information. American Journal of Psychology, 104, 311-322.

Medin, D. L., \& Edelson, S. M. (1988). Problem structure and the use of base-rate information from experience. Journal of Experimental Psychology: General, 117, 68-85.

Nelson, T. E., Biernat, M. R., \& Manis, M. (1990). Everyday base rates (sex stereotypes): Potent and resilient. Journal of Personality \& Social Psychology, 59, 664-675.

NisbetT, R, \& Ross, L. (1980). Human inference: Strategies and shortcomings of social judgment. Englewood Cliffs, NJ: Prentice-Hall.

OFIR, C. (1988). Pseudodiagnosticity in judgment under uncertainty. Organizational Behaviour \& Human Decision Processes, 42, 343-363.

REBER, A. S. (1993). Implicit learning and tacit knowledge. Oxford: Oxford University Press.

Sloman, S. A. (2000). Do frequency frames make probabilityjudgments more coherent? Paper presented at the BPS 4th International Conference on Thinking, Durham University.

Stanovich, K. E. (1999). Who is rational? Studies of individual differences in reasoning. Mahwah, $\mathrm{NJ}$ : Erlbaum.

TAYlor, S. E., \& Thompson, S. C. (1982). Stalking the elusive "vividness" effect. Psychological Review, 89, 155-181.

Thompsen, C. J., \& Borgida, E. (1996). Throwing out the baby with the bathwater? Let's not overstate the overselling of the base rate fallacy. Behavioral \& Brain Sciences, 19, 39-40.

Tversky, A., \& Kahneman, D. (1973). Availability: A heuristic for judging frequency and probability. Cognitive Psychology, 5, 207-232.

\section{NOTE}

1. The model for predicting posterior odds derived from the multiple linear regression fits is of the form

$$
\log (\hat{P})=b_{0}+b_{1} \log (Q)+b_{2} \cdot \log (L)
$$

where $\hat{P}$ is the predicted posterior odds, $Q$ the prior odds, and $L$ the likelihood ratio. We can convert this back into the original multiplicative format of Bayes's theorem, by deriving the equivalence

$$
\hat{P}=a \cdot Q^{b_{1}} \cdot R^{b_{2}}
$$

where $a=\log \left(b_{0}\right)$, or $\log$ of the intercept.

In other words, the linear weights of the regression on the log values are actually fitting the exponents of the multiplicative model. Now we can see what these weights represent by considering particular $b$ values. Suppose that the base rate is $10 \%$, so that the prior odds $Q=10 / 90$. A participant with a $b_{1}$ value of 1 would be making normative use of base rates, since they would be multiplying $(10 / 90)^{1}=10 / 90$ times the likelihood ratio. Someone with $b_{1}=0$ would have strong base-rate neglect, since $(10 / 90)^{\circ}=1$, and the posterior odds would equal the likelihood ratio. Values between 0 and 1 represent some but insufficient use of the cue. Values over 1 represent overuse of the cue. For example, if $b_{1}=2$, then $(10 / 90)^{\circ}=10 / 810$ would be the multiplier, exaggerating by squaring the true prior odds. Finally, someone with a negative value for $b_{1}$ would be showing an inverse base-rate effect in which posterior odds increase as base rate decreases. For example, with $b_{1}=-1$, the multiplier becomes $(10 / 90)^{-1}=90 / 10$. 
APPENDIX

Mean Estimates of $P(D \mid H)$ and $P(H \mid D)$ for all Societies and Faculties in Experiment 1 (H = 5 Faculty; D = 5 Society)

\begin{tabular}{|c|c|c|c|c|c|c|c|c|}
\hline \multirow[b]{2}{*}{ Society } & \multicolumn{2}{|c|}{ Engineering } & \multicolumn{2}{|c|}{ Social Science } & \multicolumn{2}{|c|}{ Arts/Humanities } & \multicolumn{2}{|c|}{ Science } \\
\hline & $P(\mathrm{D} \mid \mathrm{H})$ & $P(\mathrm{H} \mid \mathrm{D})$ & $P(\mathrm{D} \mid \mathrm{H})$ & $P(\mathrm{H} \mid \mathrm{D})$ & $P(\mathrm{D} \mid \mathrm{H})$ & $P(\mathrm{H} \mid \mathrm{D})$ & $P(\mathrm{D} \mid \mathrm{H})$ & $P(\mathrm{H} \mid \mathrm{D})$ \\
\hline Chess & 58 & 32 & 45 & 18 & 46 & 18 & 63 & 32 \\
\hline Choral & 24 & 15 & 40 & 27 & 54 & 40 & 31 & 18 \\
\hline Classical music & 36 & 18 & 52 & 24 & 70 & 39 & 48 & 19 \\
\hline Computer & 77 & 38 & 46 & 16 & 36 & 13 & 77 & 33 \\
\hline Conservative & 55 & 34 & 35 & 18 & 38 & 19 & 55 & 29 \\
\hline Drama & 31 & 11 & 58 & 28 & 76 & 47 & 34 & 14 \\
\hline Green & 33 & 15 & 66 & 33 & 62 & 29 & 53 & 22 \\
\hline Jazz music & 44 & 19 & 56 & 24 & 67 & 35 & 51 & 22 \\
\hline Judo & 53 & 29 & 47 & 22 & 48 & 23 & 45 & 26 \\
\hline Liberal Democrat & 43 & 20 & 54 & 33 & 55 & 27 & 44 & 19 \\
\hline Orienteering & 57 & 33 & 49 & 20 & 46 & 21 & 56 & 27 \\
\hline Table tennis & 53 & 29 & 46 & 23 & 45 & 22 & 50 & 27 \\
\hline Techno music & 55 & 27 & 54 & 27 & 58 & 26 & 50 & 20 \\
\hline U.F.O. & 52 & 29 & 42 & 18 & 43 & 20 & 58 & 33 \\
\hline Mean & 47.1 & 24.1 & 50.9 & 25.0 & 54.5 & 27.5 & 49.5 & 23.4 \\
\hline$S D$ & 15.1 & 8.7 & 9.2 & 6.2 & 11.6 & 9.8 & 12.0 & 5.7 \\
\hline
\end{tabular}

